\section{SAT0204 ABATACEPT SURVIVAL IN RHEUMATOID ARTHRITIS PATIENTS AT 2 YEARS IS 59\%; ITS USE AS A 2ND LINE BIOLOGIC AGENT AND LOWER BASELINE HAQ PREDICT BETTER SURVIVAL IN CLINICAL PRACTICE: A PROSPECTIVE, OBSERVATIONAL SINGLE CENTER STUDY}

I.D. Flouri ${ }^{1,1}$, A. Repa ${ }^{1}$, N. Avgoustidis ${ }^{1}$, N. Kougas ${ }^{1}$, A. Fanouriakis ${ }^{1}$, I. Papalopoulos ${ }^{1}$, C. Adamichou ${ }^{1}$, P. Kyfonidou ${ }^{1}$, E. Kampouraki ${ }^{1}$, M. Terizaki ${ }^{1}$, D.T. Boumpas ${ }^{2}$, G. Bertsias ${ }^{1}$, P. Sidiropoulos ${ }^{1} .{ }^{1}$ Rheumatology, Clinical Immunology and Allergy, Faculty of Medicine-University of Crete, Heraklion-Crete; ${ }^{2}$ 4th Internal Medicine Department, Attikon University Hospital, Athens, Greece

Background: Long-term prospective observational studies are complementary to controlled clinical trials to explore effectiveness and safety of biological therapies in clinical practice.

Objectives: To study abatacept survival, reasons of discontinuation and clinical responses in everyday clinical practice of patients with rheumatoid arthritis (RA). Methods: Prospective, observational single center study at the Rheumatology Clinic, University Hospital of Heraklion, Crete. At baseline, patient demographics, co-morbidities and disease characteristics are being recorded, while during follow-up, discontinuations, disease activity and adverse events are collected. For this analysis, all patients who received Abatacept intravenously from 6/2007 till 6/2016 were included. Kaplan-Meier curves and Cox regression analysis were used to determine drug survival and predictors thereof. Linear regression was used to compare DAS difference at 12 months between different lines of bDMARD therapy.

Results: A total of 224 patients (women: $87 \%$, seropositive: $34 \%$ ) were included. Median (IQR) age was $63(56-70)$ years, disease duration 7.4 (4-13.4) years and baseline DAS28 $5.9(5.2-6.5)$. Abatacept was the 1st bDMARD in $59(26 \%)$, 2nd in $71(32 \%)$ and $\geq 3$ rd in $94(42 \%)$ patients. During follow-up [total: 508 patient-years, median (IQR): $1.7(0.7-3.3)$ years], 54\% patients discontinued therapy (87\% for treatment failure, $10 \%$ for adverse events). Two-year treatment persistence was $59 \%$. In multivariable regression analysis, predictors of longer Abatacept survival were lower baseline HAQ [HR $(95 \% \mathrm{Cl})$ for unit increase $=2.29(1.5-3.48), \mathrm{p}<0.001]$, longer disease duration $[\mathrm{HR}(95 \% \mathrm{Cl})$ for $>8 \mathrm{vs} .<8$ years $=0.51(0.30-0.88), p=0.016]$, Abatacept as $2 n d$ vs 1 st or $\geq 3$ rd bDMARD [HR $=0.52(0.30-0.91), p=0.022]$ and a more recent year of therapy start $[H R=0.39$ $(0.16-0.95), p=0.022]$.

DAS28 $<3.2$ and remission at $6(12)$ months were achieved by $12 \%(18 \%)$ and $5 \%(7 \%)$ of patients respectively. DAS28 difference at 12 months was greater in patients who received Abatacept as the $\leq 2 n d$ than those on $\geq 3$ rd bDMARD $(p=0.009)$.

A total of 312 adverse events were registered, of which 65 were serious (SAE). Incidence of total (serious) adverse events was 61 (13)/100 patients/year. SAE included 5 cases of cancer, 10 cardiovascular events and 24 infections, mainly of the respiratory tract.

Conclusions: In the present study, Abatacept survival at 2 years was $59 \%$. The majority of patients discontinued therapy due to inadequate response. Use as a 2nd line biologic agent and lower baseline HAQ predicted better survival. Improvement in DAS was higher when Abatacept was used as the $\leq 2 n d$ bDMARD. Rates of remission or low disease activity in clinical practice are rather low, while the safety profile was excellent.

Disclosure of Interest: None declared

DOI: 10.1136/annrheumdis-2017-eular.6931

\section{SAT0205 LONG-TERM DRUG SURVIVAL AND CAUSES OF DISCONTINUATION OF SUBCUTANEOUS ABATACEPT IN RHEUMATOID ARTHRITIS: 32-MONTH RESULTS FROM A PROSPECTIVE COHORT STUDY}

J.C. Sarmiento-Monroy ${ }^{1,2}$, N. Molano-Gonzàlez ${ }^{2}$, M. Rodríguez-Jiménez $^{2}$, R.D. Mantilla ${ }^{1,2} \cdot{ }^{1}$ Rheumatology, Center of Dermatology and Rheumatology (FUNINDERMA); ${ }^{2}$ Center for Autoimmune Diseases Research (CREA), Universidad del Rosario, Bogotá D.C., Colombia

Background: Survival time or time to discontinuation of medication is a surrogate of the long-term impact on the course of disease in real life. It reflects clinical effectiveness in the absence of significant adverse events [1]. Treatment discontinuation can result from loss of efficacy or safety concerns, but prognostic factors for patient retention have not been explored thoroughly despite data for abatacept and other biologics being available from national registries [2].

Objectives: To assess drug survival of subcutaneous (SC) abatacept among patients with rheumatoid arthritis (RA) according to treatment background. Methods: This was a prospective study in which well-characterized patients with RA (by 1987 ACR criteria) were assessed from April 2014 to December 2016. Each patient was evaluated by a rheumatologist in a single rheumatology outpatient private center in Bogotá, Colombia. Patients were stratified by treatment background: $(n=54)$ biologic-naïve, $(n=24)$ switched from IV to SC abatacept administration, and $(n=51)$ inadequate response to at least 1 biologic disease-modifying antirheumatic drug. The Mantel-Haenszel test was used to test if there were any differences in the survival curves among groups. The test was performed by the survdiff function of the "survival" R package [3].

Results: A total of 129 patients were included. Baseline characteristics of patients were as follows: female gender $86 \%$, mean age $52 \pm 13$ years, median disease duration 10 (IQR 11) years, Rheumatoid Factor positive 94\%, AntiCyclic Citrullinated Peptide Antibodies 89\%, and erosive phenotype 35\%. At baseline, mean DAS28 and RAPID3 were $5.4 \pm 1.3$ and $16.6 \pm 6.8$, respectively. SC abatacept monotherapy was reported in $27 \%$. Demographics and disease characteristics were similar in all groups, except for baseline DAS28 and RAPID3 in switch group $(p<0.0001)$. According to the Mantel-Haenszel test (Fig. 1$)$, there were not significant differences between survival curves $(p=0.158)$. Forty-three patients $(33 \%)$ discontinued treatment. The most frequent reasons for drug suspension were loss of efficacy in $25 \%$, insurance-related problems (i.e., access to medication/specialist) and adverse drug reactions in 16\%. Other causes include lack of efficacy, surgeries (i.e., articular replacement), patient preference, and pregnancy.

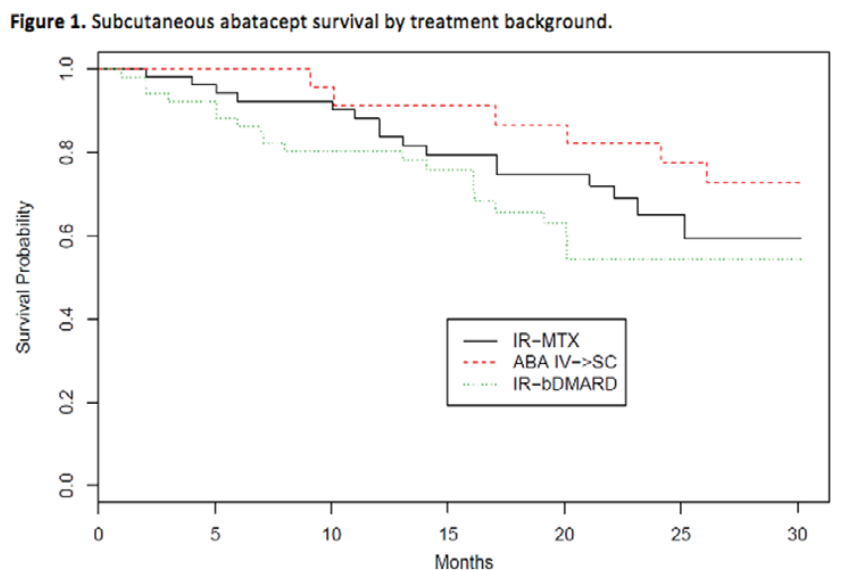

IR-MTX: inadequate response to methotrexate (biologic-naive); ABA IV $\rightarrow$ SC: switched from intravenous to subcutaneous abatacept administration (125mg-wk); IR-bDMARD: inadequate response to biologic Disease-Modifying AntiRheumatic Drugs.

Conclusions: Our results disclose a similar drug survival of SC abatacept regardless of treatment background. Patients switching from IV to SC formulation of abatacept had lower activity and functional impairment at baseline, and survival tends to be higher through follow-up. The insurance-related limitations is a reality in Latin American countries, and could have a negatively impact on survival time of several drugs.

References:

[1] Nüßlein HG, et al. BMC Musculoskelet Disord. 2015;16:176.

[2] Souto A, et al. Rheumatology (Oxford). 2016;55(3):523-34.

[3] Therneau, T. and Grambsch, P.M. 2000.

Acknowledgements: The authors are grateful to all the members of the Center of Dermatology and Rheumatology (FUNINDERMA) for their contribution to this work.

Disclosure of Interest: None declared

DOI: 10.1136/annrheumdis-2017-eular.4348

\section{SAT0206 RETENTION OF TOCILIZUMAB AS MONOTHERAPY VERSUS TNF INHIBITORS WITH CONVENTIONAL SYNTHETIC DMARDS IN RHEUMATOID ARTHRITIS PATIENTS WITH INADEQUATE RESPONSE TO TNF INHIBITORS: A STUDY FROM THE TOCERRA COLLABORATION}

K. Lauper ${ }^{1,2}$, D.C. Nordström ${ }^{3}$, K. Pavelka ${ }^{4}$, V. Hernandez ${ }^{5}$, M.J. Santos ${ }^{6}$, Z. Rotar $^{7}$, F. Iannone ${ }^{8}$, C. Codreanu ${ }^{9}$, G. Lukina ${ }^{10}$, S.L. Gale ${ }^{11}$, K. Sarsour ${ }^{11}$ A. Pethoe-Schramm ${ }^{12}$, D.S. Courvoisier ${ }^{1}$, C. Gabay ${ }^{1,2},{ }^{1}$ Univ Hosp of Geneva, Geneva; ${ }^{2}$ SCQM Registry, Zurich, Switzerland; ${ }^{3} R O B-F I N$ Helsinki Univ Central Hosp, Helsinki, Finland; ${ }^{4}$ Charles Univ, Prague, Czech Republic; ${ }^{5}$ Rheumatology Department, Hospital Clinic Barcelona, Barcelona, Spain; ${ }^{6}$ Rheumatology Unit, Hospital Garcia de Orta, Almada, Portugal:; ${ }^{7}$ BioRx.si, Univ Med Center, Ljubljana, Slovenia; ${ }^{8}$ GISEA, Univ Hospital of Bari, Bari, Italy; ${ }^{9}$ Univ of Medicine and Pharmacy, Bucharest, Romania; ${ }^{10}$ ARBITER, Inst of Rheumatology, Moscow, Russian Federation; ${ }^{11}$ Genentech, South San Francisco, CA, United States; ${ }^{12}$ F. Hoffmann-La Roche, Basel, Switzerland

Background: Tocilizumab (TCZ) as monotherapy has been shown to be more efficacious than the TNF inhibitor (TNFi) adalimumab as monotherapy in patients with rheumatoid arthritis (RA). However, effectiveness data comparing TCZ as monotherapy versus TNF inhibitors in combination with csDMARDs are limited.

Objectives: To examine retention of TCZ administered alone (TCZ mono) versus TNFi in combination with csDMARDs (TNFi combo) in patients with RA who had an inadequate response to $\geq 1 \mathrm{TNFi}$ (TNFi-IR).

Methods: Patients with RA who were TNFi-IR and treated with TCZ mono or TNFi combo with baseline (BL) data, not immediately lost to follow-up and started treatment after TCZ was available across 9 European registries in TOCERRA from 2009 to 2016 were included. The hazard for TCZ discontinuation was modeled using a country-stratified Cox proportional hazards model, adjusting for 